\title{
Impact of Violent Virtual Gaming on the Mental Standing of Youth
}

\author{
Bharath Yuvaraj R, Jayaprakash D
}

\begin{abstract}
Virtual Games, owing to their graphics, user interface, and addiction amongst youth has become a topic of discussion across the world. widely across. It is believed that only the analytical and intellectual ability of youth gets deteriorated because of virtual gaming. However, the scenario is much broader than one could believe. It traps major stages of youth ability in different aspects. Approx. four per cent of people who play games are addicted to gaming but in the case of youth, it is much severe. Mobile Gaming, Online Gaming and Virtual Dangerous Games were taken up for research on youth from different families and different age groups. The cases of leisure, parental negligence, easy access of smartphones and laptops, introvert nature of youth and its effect on youth's academic, mental and social imbalance were also considered The recent Blue whale Challenge was identified as one of all-time sinister game. It had 50 tasks to complete in which the players had to do strange tasks and ultimately kill themselves. Hundreds of players got killed but the major victim was the youth of 12-18 years age group. Owing to this youth from the same age group were considered for this research. The research also deals with a live survey on families to acquire the reason of interest in games, the chance of mental degradation, downgrading scores, crime by youth due to game influence. The research also delves into the recently popular game "Player Unknown's BattleGrounds $(P U B G)$ " and its effect on youth. several graphical representation and logs were used to support the survey.
\end{abstract}

Keywords-Blue Whale Challenge, PUBG, Virtual Gaming, and Violent \& Non-Violent Games.

\section{INTRODUCTION}

There have been several cases where virtual online gaming is considered one of the main evil causes of social imbalance especially in case of youth due to their immature mental condition. One of the main problems of mobile gaming is a distraction from the immediate environment and extreme attachment towards gaming. Many road accidents are caused by mobile phone distraction. As per the research commenced by AAA foundation on the safety of driving, $94 \%$ of youth are under a state of texting or gaming during their drive. $21 \%$ of youth encounter severe accidents because of the distraction caused by mobile games and texting. Youth are four times more prone to accidents because of using a phone while driving than adults. This shows how critical the situation is and it's alarming to control by imposing strong effect. Recent online gaming experience accelerated the scenario but due to negligence and panic, many accidents due to mobile gaming remains

Revised Manuscript Received on September 10, 2019.

Bharath Yuvaraj Ph.D. Research Scholar Department of Visual Communication, School of Mass Communication, Vels Institute of Science Technology and Advanced Studies (Deemed to be University), Chennai, Tamil Nadu, India.

Dr. Jayaprakash D Associate Professor \& Head at Department of Visual Communication, Meenakshi Academy of Higher Education and Research (Deemed to be University), Chennai, Tamil Nadu, India unreported. The analysis can differ based on different geographical and social patterns. The games, virtual games played in developed countries like USA, Russia, France etc. are not necessarily accessible in countries like Bangladesh, Pakistan or Sri Lanka due to pricing of game, high-speed internet requirement, high-end accessories and youth understanding level. There is a need for an extensive study to understand the youth of different countries with resources they have in order to analyze to what extent games affect their credibility. The research used below is based on five different parameters for a different time span of playing games on 5 youth for 1 month. This brought a reliable result which is transformed in various graphical patterns to understand its authenticity in real-time by various researchers.

The different game patterns their effect and chances were analysed wherein the games can achieve biased free verdict as good or bad. A recent surve ys on830 youth (11-17 Age group) participated from Ohio State University and the University of Amsterdam and analyzed their result that violent games really affected the academics for youth. In the conclusion section based on our research, we find the final result whether the games influence the youth anyway and what measures should be taken to overcome the issues. In our next literature survey section, we check the different researchers work and how they analyzed the information based on real-time data research on youth behavioural tendencies against gaming.

\section{LITERATURE SURVEY}

Lavinia McLean and Mark Griffiths explained how young people spend their time using screen media. Many games are designed mostly considering youth as their main consumers. Game designing companies come up with 3 major categories of consumers based on their age - 11 to 15,6 to 10 and 16 to 24 , which further differentiates their ideologies and interest. Youth in the 11 to 15 age groups generally prefer games with major special effects and challenges. 6 to 10 age groups go for intellectual and mind games and 16 to 24 go for simple but relaxing games. Then the survey indicated that male prefer to play games much longer compared to girls which reflect in their academics and extracurricular activities. The youth of the age group 8 to 11 play games 12 hours every week and 12 to 14 play 15 hours a week.

$\mathrm{He}$ also extended the positive aspects of virtual gaming. There can be a marginal benefit as well of playing games in accordance with better complexity understanding skill, better concentration on topic, a lesser distraction from the work

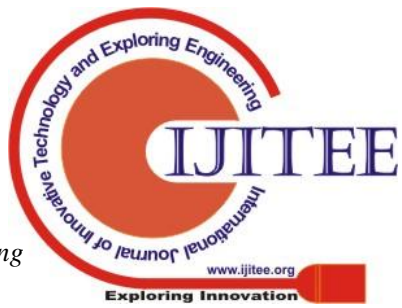


environment and learning from the challenges. They discussed the negative effect of gaming on youth as aggression, drugs, and increased vehicle accidents.

The biggest breakthrough in their research was that $89 \%$ of games designed till date have aggressive and offensive contents which do not suit the mental condition of youth. $89 \%$ is marginally a big percentage and needs immediate notice. The scenario designed for the USA might alter in different countries.

Sara Prot, Craig A. Anderson, Douglas A. Gentile, Stephanie C. Brown, and Edward L. Swing researched that games have a huge impact on the mental condition of youth. They discussed in detail that in laboratory pro-social games were encouraged to be played in order to research its effect on their mental behaviour. The results were shocking. Playing such games not only reduced aggression but also increased prosocial behaviour. Volunteers learnt many things from games. And most of them were positive ones, much to the surprise of researchers. It resulted as improvement in cooperation, helping tendency, behaviour and most importantly the ability to understand and share the feelings of another. As per their research, they extended that video games need to be analyzed in a much bigger perspective not only on positive or negative aspects. If video games are used to promote harmony and intellect then it can do miracles. On the contrary video games can have a negative effect leading to aggression, if proper conditions and situations are not used to improve the mental status of the users.

PUBG: Impact on youth and modern society of India in 2019 This research presents many information about recent PUBG gaming as the game has several pros and cons. The major pros are that it creases the ability of hand and brain to react instantly based on target's movement, increase the ability to take proper decision when the adverse situation appears, it increases the social ability when you play with friends and enhanced concentration during gameplay.

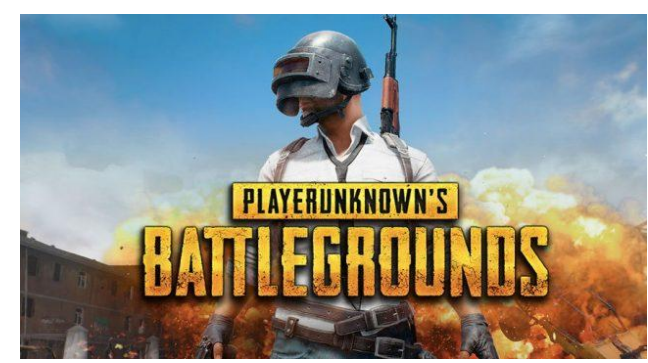

The major cons discussed were the addiction to playing the game, health issues due to long playing hours and lesser grades due to time wastage on gaming. The different aspects are available for gaming and for PUBG it shows that if you decide to play it for a lesser span of time the scenario is totally different.

The next data analysis and research contents section present the research based on different survey data and parameters on different youth for different gameplay span.

\section{DATA ANALYSIS AND RESEARCH CONTENTS}

Different researches and statistics were carried out on youth using different parameters, to identify the effect of soft and violent games for the same interval of time.

\section{Survey-based on time for nonviolent games}

A survey was done to analyze the result of time duration consumed by 5 youth on games and its impact on their performance at the scale of 100 for 1 month. The marks were decided based on 5 parameters for soft and nonviolent games. The parameters are as follows with 20 scores each.

1. Concentration

2. Aggression

3. Empathy

4. Analytical Ability

5. Stress

\begin{tabular}{|c|c|c|c|c|c|}
\hline Family / Time Spent & Youth 1 & Youth 2 & Youth 3 & Youth 4 & Youth 5 \\
\hline 10 Minutes & 93 & 90 & 86 & 80 & 89 \\
\cline { 2 - 6 } 20 Minutes & 89 & 84 & 90 & 75 & 90 \\
\cline { 2 - 6 } 30 Minutes & 90 & 87 & 86 & 71 & 85 \\
\cline { 2 - 6 } 40 Minutes & 85 & 79 & 80 & 79 & 86 \\
\cline { 2 - 6 } 50 Minutes & 79 & 80 & 79 & 82 & 84 \\
\cline { 2 - 6 } 60 Minutes & 80 & 83 & 88 & 77 & 82 \\
\cline { 2 - 6 } 70 Minutes & 85 & 78 & 85 & 72 & 85 \\
\cline { 2 - 6 } 80 Minutes & 80 & 89 & 75 & 75 & 87 \\
\cline { 2 - 6 } 90 Minutes & 83 & 82 & 78 & 73 & 86 \\
\cline { 2 - 6 }$>100$ Minutes & 81 & 80 & 81 & 77 & 79 \\
\hline
\end{tabular}

Table 1.1 - Result on the scale of 100 .

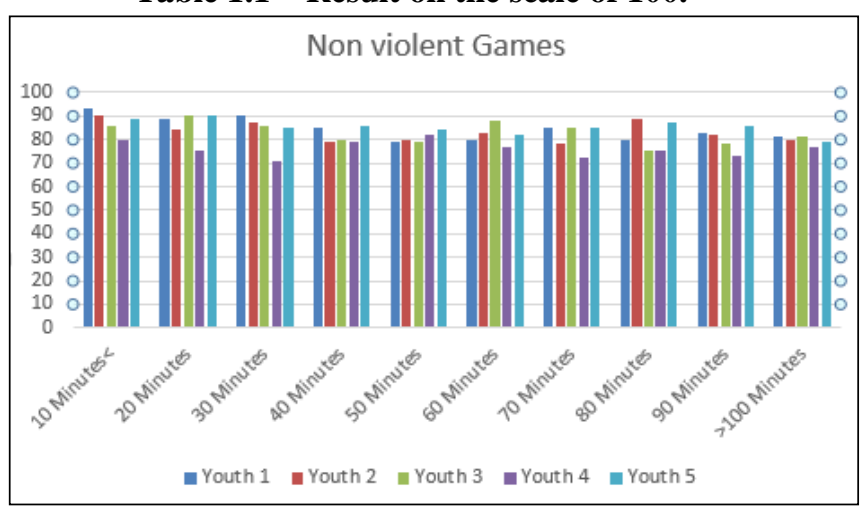

Fig. 1.1 - Result Graph 1

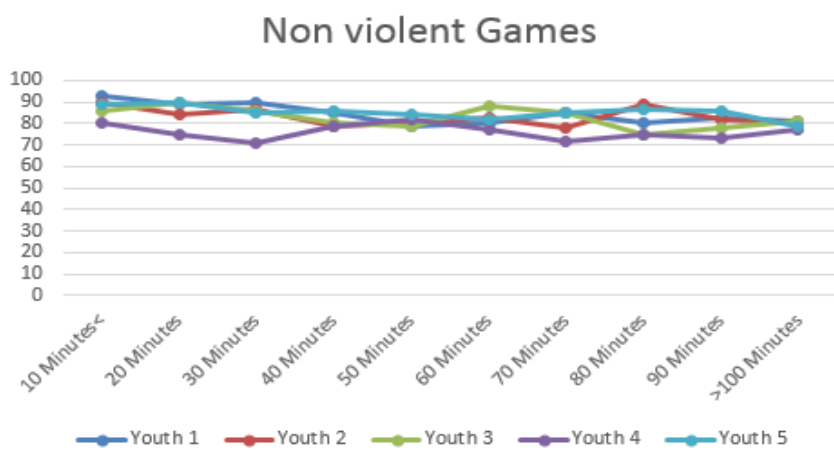

Fig. 1.2 - Result Graph 2 


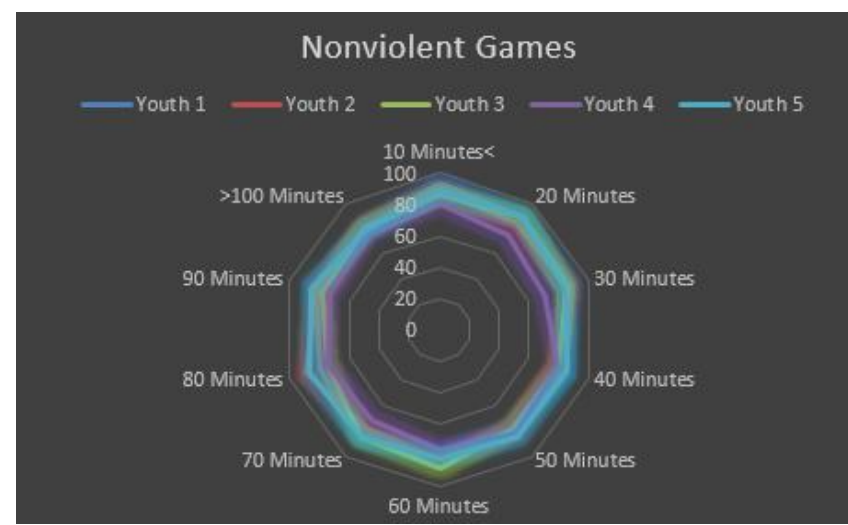

Fig. 1.3 - Result Graph 3

Survey-based on time for violent games

A survey was done to analyze the result of time duration consumed by 5 youth on games and its impact on their performance at the scale of 100 for 1 month. The marks were decided based on 5 parameters for violent games. The parameters are the same as for soft games with 20 scores each.

\begin{tabular}{|c|c|c|c|c|c|}
\hline Family / Time Spent & Youth 1 & Youth 2 & Youth 3 & Youth 4 & Youth 5 \\
\hline 10 Minutes< & 90 & 92 & 89 & 92 & 89 \\
\hline 20 Minutes & 91 & 93 & 90 & 90 & 91 \\
\hline 30 Minutes & 87 & 90 & 88 & 91 & 87 \\
\hline 40 Minutes & 86 & 85 & 87 & 90 & 86 \\
\hline 50 Minutes & 86 & 87 & 86 & 87 & 87 \\
\hline 60 Minutes & 84 & 88 & 86 & 88 & 87 \\
\hline 70 Minutes & 87 & 84 & 85 & 88 & 86 \\
\hline 80 Minutes & 83 & 83 & 84 & 89 & 85 \\
\hline 90 Minutes & 87 & 83 & 87 & 87 & 87 \\
\hline$>100$ Minutes & 84 & 82 & 83 & 89 & 89 \\
\hline
\end{tabular}

Table 1.2 - Result on the scale of 100 .

Violent Games

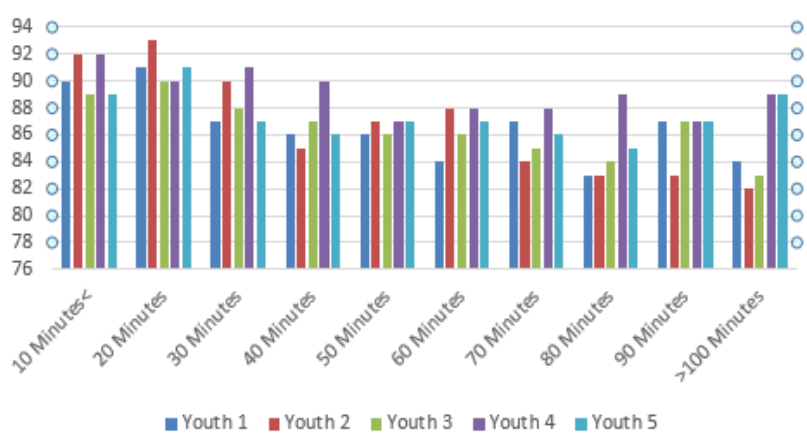

Fig. 1.4 - Result Graph 4

Violent Games

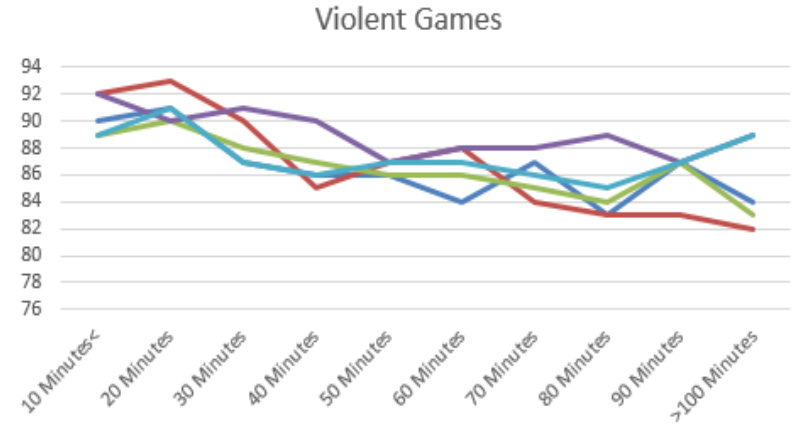

—Youth 1 -Youth $2-$ Youth $3-$ Youth $4-$ Youth 5

Fig. 1.5 - Result Graph 5

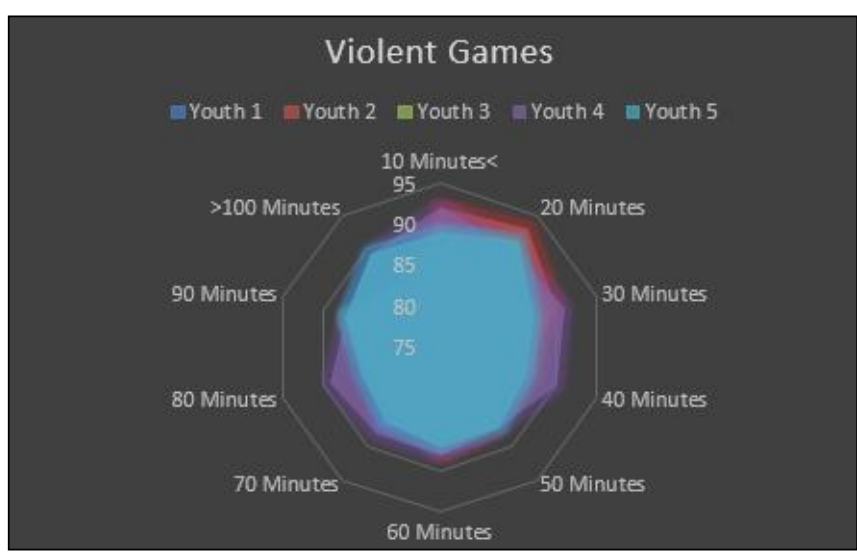

Fig. 1.6 - Result Graph 6

\section{RESULT ANALYSIS}

Two different datasets were used for 5 youth of almost same I.Q and age group with different timespans. It is evident from the survey that both kinds of games take a lot of mental space for youth. With both the games the performance decreases as shown by Fig. 1.2 and Fig. 1.4. Fig. 1.1 and Fig. 1.3 represents and supports the same information in terms of a bar graph. The radar graphs are used to easily analyze the results in Fig. 1.3 and Fig. 1.6.

In the case of nonviolent games, the situation is not much drastic. There are minor drops as per the 5 parameters Concentration, Aggression, Empathy, Analytical Ability, and Stress. In some cases, it was found for some youth that there is no change in total score and sometimes the score increases irrespective of increasing gameplay timespan. This can be due to other activities like meditation, parental behaviour or health. As per the overall result, it is clear that games certainly distract youth and play a major role in their overall performance physically and mentally.

On the contrary, violent games are much complex to analyze in terms of understanding and different youth adaptation with the game style. There were cases when survey participants from nonviolent games showed a desire to play violent games for better enjoyment and satisfaction. This explains the addictive nature of violent games and why such game companies put more violent patterns and game heroes in their virtual games.

There were several games which were too violent and obscene that it could not be used for the survey. The result available here is on acceptable games only.

As per the survey, parents should not bother about how many minutes youth play the games daily instead they should keep an eye on the games their ward selected to play.

\section{CONCLUSION}

This research paper discussed the immediate effects, parameters, evaluations and consequences of virtual gaming over the youth. The major point was drawn with the age group of 11 to 17 when the gaming matters a lot to youth and can affect their education, health and social life. The results obtained were near to reality where parents consider games to be a villain. On the contrary, there are some other results as 
well that more than games and time span a child plays the game it is important that what kind of games are being played. The children were analysed based on the games they played and the time pattern followed. Their overall performance was analysed based on 5 parameters. The result obtained depicts that violent games a re in a drastic situation than non-violent games. They hamper the youth's mentality and social credibility. Games like PUBG can be rather entertaining without hampering youth if it is played for a shorter interval of time.

\section{REFERENCES}

1. Lavinia McLean and Mark Griffiths, "The psychological effects of videogames on young people: A review" in Revista de Psicologia, Ciències de l'Educació I de l'Esport, 2013

2. Sara Prot, Craig A. Anderson, Douglas A. Gentile, Stephanie C. Brown, and Edward L. Swing, "The Positive and Negative Effects of Video Game Play" in Media and the WellBeing of Children and Adolescents (109-128). New York: Oxford University Press 2014.

3. "The radar graph" https://en.wikipedia.org/wiki/Radar_chart.

4. Marije Nije Bijvank a, Elly A. Konijn a,*, Brad J. Bushman, "We don't need no education": Video game preferences, video game motivations, and aggressiveness among adolescent boys of different educational ability levels," Journal of Adolescence by Elsevier 2011.

5. www.stillgrows.com, "PUBG: Impact on youth and modern society of India in 2019".

6. Texting and Driving Accident Statistics Available: https://www.edgarsnyder.com/car-accident/cause-ofaccident/cell-phone/cell-phone-statistics.html

7. Do bad grades + violent video games $=$ violent kids ? Available: https://www.edgarsnyder.com/car-accident/causeof-accident/cell-phone/cell-phone-statistics.html

\section{AUTHORS PROFILE}

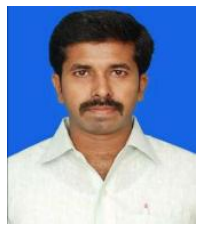

R. BharathYuvaraj M.Sc. Received his B.Sc Visual Communication under graduate degree from University of madras. M.Sc Visual Communication post graduate degree from St Peter's University, Chennai. He joined as an Assistant Professor in the Department of Visual Communication, Agurchand Manmull Jain College, Meenambakkam, Chennai. Affiliated to University of Madras, Tamil Nadu, India in the year of 2014. He has 5 years teaching experience in the Department Visual Communication. He has published several research papers in UGC Journals. His area of interest in the field of Computer Graphics, 3D Animation and Visual effects.

Dr. Jayaprakash D is an Associate Professor \& Head

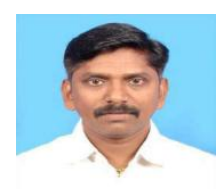
at Department of Visual Communication, Meenakshi Academy of Higher Education and Research (Deemed to be University), Chennai, India. He has done his PhD from Department of Media Sciences, Anna University, Chennai, India. He has published several research papers, a book chapter and book on various aspects of Media Studies. He specializes in Digital Cultures, Film Cultures, Broadcast Media and Communication for Development 